\title{
ANÁLISIS GENÉTICO DE RASGOS MORFOLÓGICOS DE LA SEMILLA, GERMINACIÓN Y CRECIMIENTO INICIAL EN RAULÍ (Nothofagus alpina (Poepp. \& Endl.) Oerst.) Y ROBLE (Nothofagus obliqua (Mirb.) Oerst.) EN CHILE
}

\author{
Medina, Alex ${ }^{1}$ e Ipinza, Roberto ${ }^{2}$
}

\section{RESUMEN}

Se analiza el control genético del largo y el peso de la semilla, de sus parámetros de germinación (capacidad, energía y vigor germinativo) y del desarrollo de las plántulas, en altura, durante los primeros tres meses, usando una colección de semillas de polinización abierta de 141 familias de 15 procedencias de Nothofagus alpina y 351 familias correspondientes a 38 procedencias de Nothofagus obliqua.

La heredabilidad en sentido amplio es en general moderada a alta $(0,44$ a 0,77$)$ para los rasgos físicos de la semilla y muy alta para los parámetros de germinación en $N$. alpina. Las mediciones secuenciales (seis) de altura para ambas especies muestran un importante control genético. También se observa una alta correlación en todos los pares de las variables de germinación $(0,66$ a 0,96$)$ en $N$. alpina. Las variables de crecimiento inicial en altura exhiben una alta correlación entre sí, tanto genética como fenotípica, aunque para ambas especies a medida que se desarrollan se produce una disminución de la tasa de correlación entre las mediciones iníciales y las superiores, como por ejemplo en $N$. obliqua de 0,99 a 0,51.

Palabras clave: Nothofagus, genética de peso y largo de la semilla, germinación y crecimiento.

\section{SUMMARY}

The genetic control of length, weight and germinative parameters of the seed (capacity, energy and germinative strength), along with early growth (height) during the first three years of the seedling were analyzed. The study was conduced on a complete collection of 141 and 351 open pollinated families from 15 Nothofagus alpina and 38 Nothfagus obliqua provenances, respectively. The broad sense heritability ranged from moderate to high $(0.44$ to 0.77$)$ for seed morphologic treats and very high for germinative treats in $N$. alpina. The repeated measurements analysis of height showed and important genetic control for both species.

In terms of correlations, the genetic correlation was high for all pairs of germinative parameters in N. alpina. For early growth both, genetic and phenotypic correlation, were also high between pairs of measurements; however, as seedlings growth continue the association level between earliest and latest measurement decreases in both species.

Key words: Nothofagus, seed length and weight genetics, germination and growth.

\footnotetext{
${ }^{1}$ MSc Universidad de Florida de EE.UU., Forestal Mininco S. A. Los Ángeles, Chile.

${ }^{2}$ Doctor Ingeniero de Montes, UPM de España, Instituto Forestal, Sede Valdivia. Chile. robertoipinza@infor.cl
} 


\section{INTRODUCCIÓN}

La variación genética es un conjunto de diferencias heredables que se presentan dentro de una especie o población, causada por las adaptaciones particulares de los individuos a un ambiente específico.

Si se asume que la variación geográfica o de procedencia siempre presenta un grado de control genético importante, es posible afirmar que las diferencias observadas entre poblaciones dentro de un amplio rango geográfico, siempre corresponderán a una adaptación producto de la selección natural.

Desde el punto de vista de la ordenación territorial, la variabilidad genética puede expresarse como una variación clinal, que implica cambios continuos en una gradiente ambiental y variación ecotípica que implica cambios abruptos.

En gradientes medioambientales estables, cada población local tiende a separarse por el efecto de la selección, pero por el efecto de las migraciones, o flujo de genes por polen o semilla, existe un permanente intercambio de genes con las poblaciones vecinas, es decir un proceso de homogenización, lo que tiende a mantener el gradiente genético.

Donoso (1993) establece que la producción de semilla en los árboles forestales es afectada por factores ambientales, entre ellos factores del clima, tales como radiación, temperatura, humedad, pluviometría y viento; factores del suelo como nutrientes y fertilidad; y factores bióticos como insectos, animales y enfermedades.

También existe un grado de control genético sobre la capacidad de producción de semillas, de modo que en conjunto con los efectos ambientales determinan que los árboles exhiban variabilidad en su capacidad de semillación.

Donoso (1979a; 1979b), en un estudio de 11 poblaciones de $N$. obliqua a lo largo de una gradiente latitudinal demostró una variación clinal, tanto en el tamaño de la semilla como en el número de estambres por flor masculina.

Este autor concluye también que ambos rasgos están fuertemente correlacionados con la precipitación media anual y la temperatura a lo largo de esta gradiente (Donoso, 1979b).

El peso de la semilla de Nothofagus dombeyi (Mirb.) Oerst. disminuye gradualmente a lo largo de una gradiente de norte - sur, desde 283.688 semillas por kilo en los $36^{\circ} 52^{\prime}$ LS a 497.310 semillas por kilo en los $40^{\circ} 16^{\prime}$ LS (Ordóñez, 1986).

El mayor peso de la semilla desde las poblaciones más al norte sugiere una variación ecoclinal en respuesta a las condiciones de sequedad en dirección al norte. El tamaño de la semilla y el número de estambres también aumenta desde baja a altas elevaciones (Donoso, 1996).

El presente artículo tiene como objetivo estudiar el comportamiento genético de los rasgos morfológicos de la semilla, número de semillas por kilogramo, parámetros de germinación, y crecimiento en altura bajo condiciones de invernadero, para semillas de $N$. alpina y $N$. obliqua colectadas en una gradiente latitudinal a lo largo de toda la distribución natural de estas especies en Chile. 


\section{MATERIAL Y MÉTODO}

\section{Definición de Zonas de Procedencia}

La definición de las procedencias de $N$. alpina y $N$. obliqua se ha desarrollado bajo el marco del proyecto "Mejoramiento Genético de Especies de Nothofagus de Interés Económico" $\mathrm{UACH}-I N F O R-C O N A F$ y empresas forestales.

La definición de las regiones de procedencias fue realizada por Vergara et al. (1998) usando como referencia tres macroclimas correspondientes a la división climatica de Fuenzalida (1965). Para N. obliqua se definió 14 regiones de procedencias y 39 procedencias dentro de ellas.

En el caso de $N$. alpina se determinó 7 regiones procedencias y 18 procedencias. El detalle de las procedencias de $N$. alpina y $N$. obliqua se presenta en los Cuadros $N^{\circ} 1$ y $N^{\circ} 2$.

Cuadro $\mathrm{N}^{\circ} 1$

DETALLES DE LAS PROCEDENCIAS DE $\boldsymbol{N}$. alpina

\begin{tabular}{|c|c|c|}
\hline $\begin{array}{l}\text { Regiones de } \\
\text { Procedencias }\end{array}$ & Procedencias & Punto de Colecta \\
\hline \multirow{2}{*}{$3-\mathrm{C}$} & 2. Cordillera Nahuelbuta $\left(37^{\circ} 40^{\prime}\right)$ & 2. Nacimiento \\
\hline & 3. Cholchol (3830') & 3. Pichipillahuén \\
\hline \multirow{2}{*}{$5-\mathrm{C}$} & 4. LLancacura $\left(40^{\circ} 15^{\prime}\right)$ & 4. Las Trancas \\
\hline & 5. Hueyusca $\left(40^{\circ} 55^{\prime}\right)$ & 5. Hueyusca \\
\hline \multirow{3}{*}{$8-\mathrm{A}$} & 6. Los Queñes (3510') & 6. Radal 7 Tazas \\
\hline & 7. Vilches $\left(35^{\circ} 40^{\prime}\right)$ & 7. Vilches \\
\hline & 8. Embalse Bullileo (36익) $750 \mathrm{msnm}$ & 8. Embalse Bullileo \\
\hline \multirow{3}{*}{$9-A$} & 9. Recinto (3650') $700 \mathrm{msnm}$ & 9. Recinto \\
\hline & 10. Santa Barbara (37웅' $500 \mathrm{msnm}$ & 10. Santa Bárbara \\
\hline & 11. Jauja (3840') 650 msnm. & 11. Jauja \\
\hline \multirow{3}{*}{$11-\mathrm{A}$} & 12. Malalcahuello $\left(38^{\circ} 5^{\prime}\right) 1000 \mathrm{msnm}$ & 12. Malalcahuello \\
\hline & 13. Melipeuco $\left(38^{\circ} 50^{\prime}\right)$ & 13. Melipeuco \\
\hline & 14. Curarrehue $\left(39^{\circ} 50^{\prime}\right)$ & 14. Curarrehue \\
\hline $13-\mathrm{A}$ & 15. Releco $\left(39^{\circ} 40^{\prime}\right)$ & 15. Releco \\
\hline
\end{tabular}




\section{Cuadro $\mathrm{N}^{\circ} 2$}

DETALLES DE LAS PROCEDENCIAS DE $\boldsymbol{N}$. obliqua

\begin{tabular}{|c|c|c|}
\hline $\begin{array}{c}\text { Región de } \\
\text { Procedencias }\end{array}$ & Procedencias & Lugar de cosecha \\
\hline \multirow{2}{*}{$1-C$} & 1. Cerro el Roble ( $\left.33^{\circ} 07^{\prime}\right)$ & Tiltil \\
\hline & 3. Loncha ( $34^{\circ} 12^{\prime}$ ) & Alhue \\
\hline \multirow{4}{*}{$2-C$} & 4. Estero Nilahue $\left(34^{\circ} 30^{\prime}\right)$ & Alto Colorado \\
\hline & 5. Empedrado ( $35^{\circ} 30^{\prime}$ ) & Reserva Nacional Los Ruiles \\
\hline & 6. $\quad$ Ninhue $\left(36^{\circ} 20^{\prime}\right)$ & Ninhue \\
\hline & & Quirihue \\
\hline \multirow{4}{*}{$3-\mathrm{C}$} & 7. Cerrro Cayumanqui ( $36^{\circ} 40^{\prime}$ ) & Cayumanqui \\
\hline & 8. $\quad$ Cordillera Nahuelbuta ( $\left.37^{\circ}-40^{\prime}\right)$ & Curanilahue \\
\hline & 9. Lago Lanalhue ( $37^{\circ} 55^{\prime}$ ) & Lago Lanalhue \\
\hline & 10. Cholchol ( 38우 30 ) & Pichipillahuén \\
\hline \multirow{2}{*}{$4-C$} & 11. Cuesta Lastarria ( 3910 10 ) & Lastarria \\
\hline & 12. Cruces ( $\left.39^{\circ} 35^{\prime}\right)$ & Cayumapu (Cruces) \\
\hline \multirow{3}{*}{$5-\mathrm{C}$} & 13. Llancacura ( $\left.40^{\circ} 15^{\prime}\right)$ & LLancacura \\
\hline & 14. Rio Negro $\left(40^{\circ} 50^{\prime}\right)$ & Rio Negro \\
\hline & 15. Fresia $\left(41^{\circ} 15^{\prime}\right)$ & Purranque \\
\hline \multirow{2}{*}{$6-\mathrm{D}$} & 16. Victoria (38ำ $\left.10^{\prime}\right)$ & Victoria \\
\hline & 17. Quepe ( $\left.38^{\circ} 55^{\prime}\right)$ & Quepe \\
\hline \multirow{3}{*}{$7-\mathrm{D}$} & 18. Malalhue ( $\left.39^{\circ} 30^{\prime}\right)$ & Malalhue \\
\hline & 19. Puerto Nuevo ( $40^{\circ} 10^{\prime}$ ) & Futrono \\
\hline & 20. Hacienda Rupanco (405 5' ) & Rupanco \\
\hline \multirow{5}{*}{$8-\mathrm{A}$} & 21. Cord. Colchagua (3430`) $2.000 \mathrm{msnm}$ & Sierras de Bellavista \\
\hline & 22. Altos de Vilches ( $35^{\circ} 40^{\prime}$ ) $2.000 \mathrm{msnm}$ & Alto Lircay \\
\hline & 23. Corrales ( $\left.36^{\circ} 20^{\prime}\right) 1.400 \mathrm{msnm}$ & Altos de Vilches \\
\hline & 24. Vilches ( $35^{\circ} 40^{\prime}$ ) $800 \mathrm{msnm}$ & Vilches \\
\hline & 25. Embalce Bullileo (36을 20 750 msnm & Bullileo \\
\hline \multirow{4}{*}{$9-A$} & 26. R. Nacional Ñuble $\left(37^{\circ} 00^{\prime}\right) 1.200 \mathrm{msnm}$ & Reserva Nacional Ñuble \\
\hline & 27. R. Nacional Ralco (37은 $1.000 \mathrm{msnm}$ & Ralco \\
\hline & 28. Recinto ( $36^{\circ} 50^{\prime}$ ) $700 \mathrm{msnm}$ & Recinto \\
\hline & 29.Santa Bárbara ( 37ㅇ4우 ) 500 msnm & Santa Bárbara \\
\hline \multirow{4}{*}{$11-\mathrm{A}$} & 31. Lago Galletue ( $38^{\circ} 40^{\prime}$ ) & Galletue \\
\hline & 32. Cunco ( $\left.38^{\circ} 50^{\prime}\right)$ & Cunco \\
\hline & 33. Lago Colico ( $39^{\circ} 05^{\prime}$ ) & Colico \\
\hline & 34. Curarrehue ( $\left.39^{\circ} 25^{\prime}\right)$ & Curarrehue \\
\hline \multirow{2}{*}{$13-A$} & 37. Choshuenco ( $\left.39^{\circ} 50^{\prime}\right)$ & Choshuenco \\
\hline & 38. Llifén ( $\left.40^{\circ} 10^{\prime}\right)$ & Llifen \\
\hline
\end{tabular}




\section{Colecta de Semilla}

La colecta de semilla fue realizada entre los meses de febrero y marzo de 1999. Se colectó semilla de 35 y 14 procedencia para $N$. obliqua y $N$. alpina, respectivamente, faltando para ambas especies las procedencias localizadas en Argentina. Para cada procedencia, se cosechó semilla en 10 individuos, completando así 351 familias de medios hermanos para $N$ alpina y 141 familias para $N$. obliqua, con totales de $19,23 \mathrm{~kg}$ y $1,88 \mathrm{~kg}$ de semilla, respectivamente. Las actividades de colecta, procesamiento y almacenaje de la semilla fueron realizadas por personal del laboratorio de semilla del Instituto Forestal, Sede Concepción.

\section{Análisis de Semilla y Crecimiento Inicial}

Para identificar los patrones de variación genética reflejados en la semilla y el crecimiento inicial de las poblaciones naturales de N. alpina y $N$. obliqua Medina (2000), se realizó cuatro tipos de análisis:

Tamaño y peso individual de muestras de semilla.

Número de semillas por kilogramo

Parámetros germinativos

Crecimiento inicial en altura en condiciones de invernadero

\section{-Tamaño y Peso Individual de Muestras de Semilla}

Se midió el peso individual ( $\mathrm{g}$ y el largo individual $(\mathrm{mm})$ de una muestra de semillas de cada una de los árboles considerados en la colección. Cada muestra consistió de 30 semillas tomadas al azar, es decir que cada árbol tiene 30 replicas.

\section{-Número de Semillas por Kilogramo}

La determinación del número de semillas por kilo se efectuó de acuerdo con las normas establecidas por el ISTA (1963), implementando ligeras modificaciones con relación al tamaño de la muestra, debido a limitaciones en la cantidad de semilla disponible para análisis. Se tomaron al azar cuatro réplicas de 50 semillas de cada árbol. Cada réplica se peso individualmente y luego se extrapoló dicho valor al número de semillas por kilogramo.

\section{-Parámetros Germinativos}

Para este análisis se consideró solo la especie $N$. alpina. Se contemplan los parámetros germinativos propuestos por Harmann y Kester (1975); Chaisurisri et al. (1992) y El-Kassaby et al. (1992). Los parámetros se definen como sigue:

Capacidad Germinativa (CG): Porcentaje de semillas germinadas al final del período de evaluación.

Energía germinativa (EG): Corresponde al máximo cociente obtenido al dividir el porcentaje de germinación diaria acumulada por el correspondiente número de días, en otras palabras es la germinación media diaria de los componentes más vigorosos del lote de semillas. Matemáticamente se expresa como la primera derivada de la curva de germinación acumulada.

Valor de Germinación (VG): Este parámetro corresponde al producto entre la germinación media diaria (GMD), que es el porcentaje de germinación final dividido por el total de días de control (34 días), y el Valor Cima (VC), que corresponde al porcentaje de germinación 
en el punto de máxima germinación marginal $(\mathrm{T})$, dividido por el número de días transcurridos.

$$
\begin{array}{lll}
\mathrm{VG}=\mathrm{GMD}{ }^{*} \mathrm{VC} & \\
\text { Donde: } & \mathrm{VG} & =\text { Valor de germinación } \\
\mathrm{GMD} & =\text { Germinación media diaria } \\
\mathrm{VC} & =\text { Valor cima }
\end{array}
$$

En la Figura $\mathrm{N}^{\circ} 1$ se puede apreciar el punto $\mathrm{T}$, donde la razón de germinación comienza a decrecer, y corresponde al punto donde se alcanza el Valor Cima. Por su parte el punto $G$ marca el porcentaje de germinación acumulada final, alcanzado por la muestra al término del ensayo.

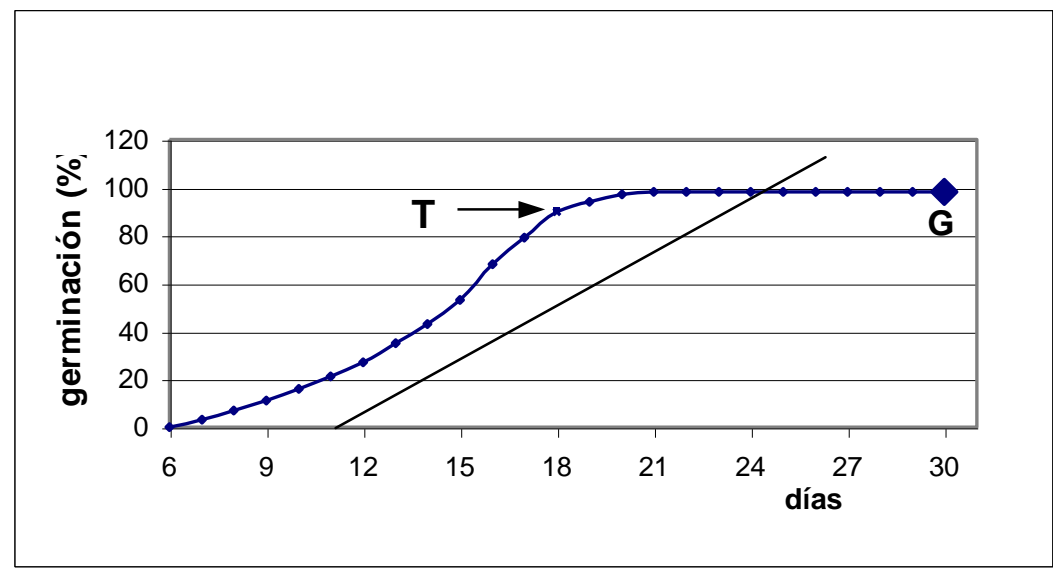

T corresponde al porcentaje de germinación en el punto de máxima germinación marginal. El punto $\mathrm{G}$ es el porcentaje de germinación acumulada final, alcanzado por la muestra al término del ensayo.

\section{Figura $\mathrm{N}^{\circ} 1$}

\section{CURVA DE GERMINACIÓN TÍPICA DE UNA MUESTRA DE SEMILLAS GERMINADAS}

Para determinar los parámetros de germinación de cada familia, se utilizó tres réplicas de 104 semillas cada una. De acuerdo con lo sugerido por Chaisurisri et al. (1992), para normalizar las variables respuesta y verificar la homogeneidad de varianza, se transformó el valor de CG a unidades de Bliss. Los parámetros EG y VG no se transformaron.

\section{-Viverización}

Todas las actividades de la viverización se desarrollaron bajo la metodología definida y aplicada por la empresa Mininco S A para las especies N. alpina y N. obliqua (Rodríguez y Medina, 2000). 
La siembra fue realizada durante el mes de septiembre del año 1, para de esta forma obtener plantas a finales de junio del año 2, con un tamaño variable entre 35 y $60 \mathrm{~cm}$ de altura y un diámetro de cuello entre 4 y $8 \mathrm{~mm}$.

Debido a la gran cantidad de familias y progenies la siembra comenzó en la segunda semana de septiembre de 1999, extendiéndose hasta la tercera semana de octubre.

\section{-Crecimiento Inicial en Altura Bajo Condiciones de Invernadero}

Se realizaron 6 controles de altura, cada 15 días, para llevar un control del ritmo de crecimiento en altura de la planta. Las mediciones se realizaron con una regla graduada desde el cuello hasta el ápice de la planta y los registros se expresaron en milímetros.

El número de replicas considerado para $N$. alpina fue de 30 y para $N$. obliqua de 10.

\section{Análisis Genético}

El análisis genético se realizó mediante el programa ASREML (Gilmour et al. 2000), donde se ajusta el siguiente modelo general mixto:

$$
y=\mathrm{X} \beta+\mathrm{Zu}+e
$$

Donde: $\quad y$ es el vector de observación

$X$ es la matriz de diseño para los efectos fijos $\beta$

$Z$ es la matriz de diseño para los efectos aleatorios $u \sim\left(0, \sigma^{2} \mathrm{G}\right)$

$e \sim\left(0, \sigma^{2} \mathrm{R}\right)$ es un vector de residuos.

En algunos casos $\sigma^{2}$ es incorporada en $R$ y G. ASREML permite que tanto Gy Rse definan como la suma directa del producto directo de las matrices de covarianza parametrizadas. En el caso del análisis genético se utiliza la matriz de parentesco en la definición de G

De acuerdo a Gilmour et al. (2000), para un G y R dado, las soluciones para $\beta$ y $u$ se obtiene resolviendo las ecuaciones del modelo mixto.

La estimación de los parámetros de varianzas son determinados maximizando la función de máxima verosimilitud restringida (REML) usando el algoritmo de información promedio.

\section{Modelo Clonal}

Para el tamaño y peso individual de la semilla se aplicó el modelo clonal, sugerido por Chaisurisri et al. (1992), donde el efecto aleatorio es el clon o variación entre árboles.

El modelo se resolvió mediante una estrategia en dos fases, primero un análisis univariado para estimar los componentes de varianza inicial y luego un análisis bivariado propiamente tal. Este se aplicó para cada una de las especies.

Los parámetros genéticos determinados son la heredabilidad clonal $\left(\mathrm{H}^{2}\right.$ clonal $)$ en sentido amplio (Chaisurisri et al., 1992; El-Kassaby et al., 1992), con su respectivo error estándar, también se establecen las correlaciones genéticas $\left(r_{\text {genética }}\right)$, correlación residual $\left(r_{\text {error }}\right)$ 
y la correlación fenotípica ( $r_{\text {fenotípica }}$ ) entre las dos variables con sus respectivos errores estándares.

$$
\begin{aligned}
& \mathrm{H}_{\text {clonal }}^{2}=\sigma_{\text {clonal }}^{2} / \sigma_{\text {fenotípica }}^{2} \\
& \text { Donde: } \sigma_{\text {fenotípica }}^{2}=\sigma_{\text {clonal }}^{2}+\sigma_{\text {error }}^{2} \\
& \sigma^{2} \text { clonal es la varianza entre clones o árboles y } \\
& \sigma^{2} \text { error es la varianza del error }
\end{aligned}
$$

Las correlaciones entre rasgos $\left(r_{\text {genética }}, r_{\text {error }}\right.$ y la $\left.r_{\text {fenotípica }}\right)$ se estiman a partir de

$$
r=\operatorname{cov}(x, y) /\left(\sigma_{x}{ }^{*} \sigma_{y}\right)
$$

Donde: $\operatorname{cov}(x, y)$ es la covarianza entre el rasgo $x$ e $y$, respectivamente.

$$
\sigma_{x}^{2} \text { e } \sigma_{y}^{2} \text { son las varianza para } x \text { e } y \text {, respectivamente. }
$$

Detalles sobre esta estimación pueden encontrarse en Burdon et al. (1992) e Ipinza et al. (1994). Los errores estándares se estiman de acuerdo a Gilmour, et al. (2000).

Para el número de semillas por kilogramo se aplicó el modelo clonal mediante un análisis univariado para cada especie. En este caso se estimo la $\mathrm{H}^{2}$ clonal con su respectivo error estándar.

Para la estimación de los parámetros genéticos asociados a los parámetros germinativos, CG, EG y VG, también se utilizó el modelo clonal, mediante una estrategia en dos fases, primero un análisis univariado y luego un análisis trivariado.

Este análisis se aplicó solo para la especie $N$. alpina. Al igual que en el primer análisis se estimo las $\mathrm{H}^{2}$ clonal, $r_{\text {genética, }} r_{\text {error }}$ y la $r_{\text {fenotípica }}$ entre las variables $C G$, EG y VG con sus respectivos errores estándares.

\section{Modelo Familiar}

Las variables crecimiento en altura fueron analizadas mediante un modelo familiar, de medio hermanos, con dos efectos aleatorio; la familia y la procedencia.

Para su solución se utilizó el programa ASREML (Gilmour, et al. 2000).

El análisis se realizó en una estrategia en tres fases, primero un análisis univariado y luego un análisis multivariado para obtener los valores iniciales para así comenzar la iteración del análisis multivariado final.

El análisis para las seis mediciones de altura, de cada especie corresponde al de mediciones repetidas (Gilmour, et al. 2000). En este caso se estimo las $h^{2}, r_{\text {genética }}, r_{\text {error }}$ y la $r_{\text {fenotípica }}$ entre las variables altura $a_{i}$ donde $i=1 \ldots 6$, son sus respectivos errores estándares. 


\section{RESULTADOS}

\section{Tamaño y Peso Individual de Muestras de Semilla}

En el Cuadro $\mathrm{N}^{\circ} 3$ se muestra las heredabilidades en sentido amplio para el largo y peso individual de la semilla entre árboles de $N$. alpina y $N$. obliqua, respectivamente.

\section{Cuadro $\mathrm{N}^{\circ} 3$ \\ $H^{2}$ PARA EL LARGO Y PESO INDIVIDUAL DE LA SEMILLA ENTRE ARBOLES DE $N$. alpina Y N. obliqua}

\begin{tabular}{|l|c|c|}
\hline Heredabilidad Clonal & N. alpina & N. obliqua \\
\hline $\mathrm{H}^{2}$ largo \pm ee & $0,5483 \pm 0,0319$ & $0,7736 \pm 0,0139$ \\
\hline $\mathrm{H}^{2}$ peso $\pm \mathrm{ee}$ & $0,4483 \pm 0,0322$ & $0,5785 \pm 0,0197$ \\
\hline
\end{tabular}

ee: Error Estandar

La $\mathrm{H}^{2}$ para el largo y peso de la semilla de $N$. alpina, determinada por la variación entre árboles, puede ser considerada como alta, es decir el efecto clonal es importante, demostrando con ello un fuerte control genético, y por lo tanto estos rasgos se encuentran fijados entre árboles.

El menor control relativo del peso puede deberse a una mayor variabilidad por factores ambientales, probablemente presencia de semilla vana.

Para N. obliqua, las heredabilidades clonales del largo y peso individual de la semilla, determinadas por la variación entre árboles, son mayores que $N$. alpina, pudiendo ser catalogadas como altas.

En el Cuadro $N^{\circ} 4$ se muestra la correlación genética, residual y fenotípica entre árboles de $N$. alpina y $N$. obliqua en función del largo y peso individual de la semilla.

\section{Cuadro $\mathrm{N}^{\circ} 4$}

CORRELACIONES GENÉTICAS, RESIDUAL Y FENOTÍPICA ENTRE EL LARGO

Y EL PESO INDIVIDUAL DE LA SEMILLA ENTRE ARBOLES DE $N$. alpina y $N$. obliqua

\begin{tabular}{|l|c|c|}
\hline Correlaciones & N. alpina & N. obliqua \\
\hline$r_{\text {genética, largo*peso } \pm \text { ee }}$ & $0.4363 \pm 0.0730$ & $0,8168 \pm 0,0190$ \\
\hline$r_{\text {residual, largo*peso } \pm \text { ee }}$ & $0,3257 \pm 0,0144$ & $0,2336 \pm 0,0096$ \\
\hline$r_{\text {fenotípica, largo*peso } \pm \text { ee }}$ & $0,3789 \pm 0,0380$ & $0,6186 \pm 0,0200$ \\
\hline
\end{tabular}

ee: Error Estándar

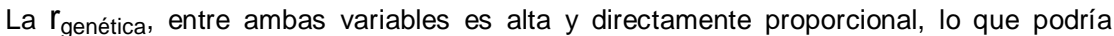
sugerir que hay un efecto clonal, es decir, existe una correlación genética entre árboles que originan semillas pequeñas y livianas y otros árboles con semilla pesada y más grande.

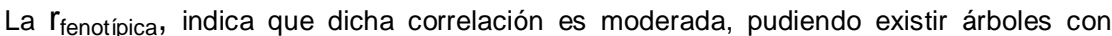
semilla fenotípicamente muy pesada y pequeña o viceversa semilla grande y liviana. 
La tendencia natural es que semilla más grande sea más pesada, pero existe una ligera influencia externa determinada por la incidencia de las perforaciones ocasionadas por el micro lepidóptero Perzelia spp. que altera en parte esta relación.

La $r_{\text {genética }}$ y la $r_{\text {fenotípica }}$ son altas y directamente proporcionales, lo que en definitiva establece que tanto desde el punto de vista genético como del fenotípico existe una alta relación y que esta no es al azar.

En términos genéticos esta relación es más sólida que en $N$. alpina. La $r_{\text {residual muestra }}$ que existe una baja a moderada autoregresividad entre ambas variables.

La mayor correlación fenotípica observada en $N$. obliqua obedece a que la variación entre árboles, localizados en un amplio espectro latitudinal, obedece a efectos genéticos. Es decir la variación fenotípica es un buen estimador de la variación genética, lo que tiene muchas implicancias prácticas.

En relación a $N$. alpina existe una mayor variación ambiental provocada por el efecto distorsionador del daño provocado por Perzelia spp., esta tiene una incidencia menor en $N$. obliqua que en $N$. alpina.

\section{Número de Semillas por Kilogramo}

En el Cuadro $\mathrm{N}^{\circ} 5$ se muestra las heredabilidades en sentido amplio para el número de semillas por kilogramo entre árboles de $N$. alpina y $N$. obliqua.

\section{Cuadro $\mathrm{N}^{\circ} 5$ \\ $H^{2}$ PARA EL NUMERO DE SEMILLAS POR KILOGRAMO ENTRE ARBOLES \\ PARA N. alpina y N. obliqua}

\begin{tabular}{|c|c|}
\hline Especie & $\mathbf{H}^{2}$ semillas por kilo \pm ee \\
\hline N. alpina & $0,9394 \pm 0,0081$ \\
\hline N. obliqua & $0,9295 \pm 0,0060$ \\
\hline
\end{tabular}

ee: Error Estándar

La $\mathrm{H}^{2}$ del número de semillas por kilogramo entre los árboles refleja en ambas especie un valor muy alto, con una escasa influencia de factores ambientales, denotando por lo mismo un elevado nivel de control genético.

Esto podría sugerir la utilización de este rasgo simple para identificar la procedencia de origen de un lote de semilla.

\section{Parámetros Germinativos}

En el Cuadro $N^{\circ} 6$ se muestra las heredabilidades en sentido amplio para la Capacidad Germinativa (CG), Energía Germinativa (EG) y Valor de Germinación (VG) entre árboles de N. alpina.

La $\mathrm{H}^{2} \mathrm{CG}$ es muy alta, $\mathrm{H}^{2} \mathrm{EG}$ es moderada y $\mathrm{H}^{2} \mathrm{VG}$ es baja. Las tres heredabilidades en sentido amplio reflejan que el efecto clonal es importante y que explica la gran variación observada 
en la capacidad germinativa, energía germinativa y en menor nivel el valor germinativo entre los distintos progenitores. El efecto clonal es bajo para el valor de germinación (VG).

\section{Cuadro $\mathrm{N}^{\circ} 6$ \\ $H^{2}$ PARA LA CG, EG Y VG ENTRE ARBOLES DE N. alpina}

\begin{tabular}{|c|c|}
\hline Heredabilidad Clonal & Valor \pm ee \\
\hline$H^{2}{ }_{C G}$ & $0,9049 \pm 0,0160$ \\
\hline$H^{2}{ }_{E G}$ & $0,2575 \pm 0,0699$ \\
\hline$H^{2}{ }$ & $0,1564 \pm 0,0679$ \\
\hline
\end{tabular}

ee: Error Estándar

En el Cuadro $N^{\circ} 7$ se muestra la correlación genética, residual y fenotípica de la CG, EG y VG entre árboles de $N$. alpina.

Las correlaciones genéticas y fenotípicas entre la capacidad germinativa y la energía germinativa son de moderadas a altas e inversamente proporcionales. En cambio, la relación genética y fenotípica entre la $C G^{*} V G$ es prácticamente nula y su error estándar es relativamente alto. La correlación genética entre $E G^{*} V G$ es alta, pero la fenotípica es muy baja, ambas están inversamente relacionadas, también es importante hacer notar que el error estándar de la correlación genética y fenotípica es muy alto.

El valor pequeño de la $\mathrm{H}^{2}$ de VG y su alto error estándar indican que es un parámetro de fuerte efecto ambiental entre árboles.

Cuadro $\mathrm{N}^{\circ} 7$

CORRELACIONES GENÉTICAS, RESIDUAL Y FENOTÍPICA DE LA CG, EG Y VG ENTRE ARBOLES DE N. alpina

\begin{tabular}{|c|c|}
\hline Correlaciones & Valores \pm ee \\
\hline$r_{\text {genética, }} \mathrm{CG}^{\star} \mathrm{EG}$ & $-0,4824 \pm 0,1355$ \\
\hline$r_{\text {genética, }} \mathrm{CG}^{*} \mathrm{VG}$ & $0,0955 \pm 0,1854$ \\
\hline$r_{\text {genética, }} \mathrm{EG}^{*} \mathrm{VG}$ & $-0,3286 \pm 0,2673$ \\
\hline$r_{\text {residual, }} \mathrm{CG}^{*} \mathrm{EG}$ & $0,1100 \pm 0,0751$ \\
\hline$r_{\text {residual, }} \mathrm{CG}^{*} \mathrm{VG}$ & $-0,0198 \pm 0,0756$ \\
\hline$r_{\text {residual, }} E^{*} V G$ & $-0,0116 \pm 0,0749$ \\
\hline$r_{\text {fenotípica, }} \mathrm{CG}^{*} \mathrm{EG}$ & $-0,2036 \pm 0,0702$ \\
\hline$r_{\text {fenotípica, }} C G^{*} V G$ & $0,0303 \pm 0,0701$ \\
\hline$r_{\text {fenotípica, } E G^{*} V G}$ & $-0,0751 \pm 0,0624$ \\
\hline
\end{tabular}

ee: Error Estándar 


\section{Crecimiento Inicial en Altura en Condiciones de Invernadero}

\section{-Nothofagus alpina}

En el Cuadro $\mathrm{N}^{\circ} 8$ se muestra las heredabilidades, en sentido restringido o individual, para cada una de las seis mediciones de altura de las progenies de N. alpina.

\section{Cuadro $\mathrm{N}^{\circ} 8$}

$H^{2}$ PARA CADA UNA DE LAS SEIS MEDICIONES DE ALTURA DE LAS PROGENIES DE N. alpina EN CONDICIONES DE INVERNADERO

\begin{tabular}{|c|c|}
\hline Rasgos & Heredabilidad individual \pm ee \\
\hline$h^{2}{ }_{1}$ & $0,3398 \pm 0,0451$ \\
\hline$h^{2}{ }_{2}$ & $0,6401 \pm 0,0494$ \\
\hline$h^{2}{ }^{2}$ & $0,6960 \pm 0,0769$ \\
\hline$h^{2}{ }_{h}$ & $0,7921 \pm 0,1029$ \\
\hline$h^{2}{ }_{h 5}$ & $0,8416 \pm 0,1275$ \\
\hline$h^{2}{ }_{h 6}$ & $0,8530 \pm 0,1424$ \\
\hline
\end{tabular}

ee: Error Estándar

El crecimiento en altura de las plantas de las distintas familias de $N$. alpina exhibe un control genético creciente desde moderado $\left(h^{2}{ }_{h 1}=0,33\right)$ a alto $\left(h_{h 6}^{2}=0,85\right)$ y también se incrementan levemente sus respectivos errores estándares.

En el Cuadro $\mathrm{N}^{\circ} 9$ se muestra la correlación genética entre las seis mediciones de altura de la progenie de $N$. alpina

\section{Cuadro $\mathrm{N}^{\circ} 9$}

CORRELACIONES GENÉTICAS ENTRE LAS MEDICIONES DE ALTURA DE LAS PROGENIES DE N. alpina EN CONDICIONES DE INVERNADERO

\begin{tabular}{|c|c|c|c|c|c|c|}
\hline $\begin{array}{c}\mathbf{r}_{\text {genética }} \\
(\mathbf{x}, \mathbf{y})\end{array}$ & $\mathbf{h}_{\mathbf{1}} \pm \mathbf{e e}$ & $\mathbf{h}_{\mathbf{2}} \pm \mathbf{e e}$ & $\mathbf{h}_{\mathbf{3}} \pm \mathbf{e e}$ & $\mathbf{h}_{\mathbf{4}} \pm \mathbf{e e}$ & $\mathbf{h}_{\mathbf{5}} \pm \mathbf{e e}$ & $\mathbf{h}_{\mathbf{6}} \pm \mathbf{e e}$ \\
\hline $\mathrm{h}_{1}$ & - & & & & & \\
\hline $\mathrm{h}_{2}$ & 0,8683 & - & & & & \\
\hline $\mathrm{h}_{3}$ & 0,7167 & 0,9428 & - & & & \\
& $\pm 0,0281$ & $\pm 0,0077$ & & & & \\
\hline $\mathrm{h}_{4}$ & 0,6253 & 0,8650 & 0,9562 & - & & \\
& $\pm 0,0245$ & $\pm 0,0071$ & $\pm 0,0000$ & & & \\
\hline \multirow{2}{*}{$h_{5}$} & 0,5095 & 0,7507 & 0,8768 & 0,9682 & & \\
& $\pm 0,0200$ & $\pm 0,0061$ & $\pm 0,0000$ & $\pm 0,0000$ & & \\
\hline \multirow{2}{*}{$h_{6}$} & 0,4179 & 0,6427 & 0,7859 & 0,9123 & 0,9797 & \\
& $\pm 0,0164$ & $\pm 0,0053$ & $\pm 0,0000$ & $\pm 0,0000$ & $\pm 0,0000$ & \\
\hline
\end{tabular}

ee: Error Estándar 
La $r_{\text {genética }}$ desde $h_{1}{ }^{*} h_{2}$ a $h_{1} * h_{6}$ disminuye monótonamente $(0,86$ a 0,41$)$, sugiriendo una menor correlación edad-edad, desde alta a moderada. Considerando esta tendencia, en que decrece la correlación edad-edad en mediciones sucesivas efectuadas aproximadamente cada 15 días, se puede presumir una correlación muy baja entre plantas y árboles adultos, situación que podría impedir una selección precoz en función de la característica altura, a menos que se estabilice dicha relación antes de salir las plantas del invernadero.

En el Cuadro $\mathrm{N}^{\circ} 10$ se muestra la correlación fenotípica entre las seis mediciones de altura de la progenie de $N$. alpina.

$$
\text { Cuadro } \mathrm{N}^{\circ} 10
$$

CORRELACIONES FENOTÍPICAS ENTRE LAS MEDICIONES DE ALTURA DE LA PROGENIE DE N. alpina EN CONDICIONES DE INVERNADERO

\begin{tabular}{|c|c|c|c|c|c|c|}
\hline $\begin{array}{c}\mathbf{r}_{\text {fenotípico }} \\
(x, y)\end{array}$ & $h_{1} \pm$ ee & $h_{2} \pm$ ee & $h_{3} \pm \mathbf{e e}$ & $h_{4} \pm \mathrm{ee}$ & $h_{5} \pm e e$ & $h_{6} \pm$ ee \\
\hline $\mathrm{h}_{1}$ & - & & & & & \\
\hline $\mathrm{h}_{2}$ & $\begin{array}{c}0,8335 \\
\pm 0,0217\end{array}$ & - & & & & \\
\hline $\mathrm{h}_{3}$ & $\begin{array}{c}0,5767 \\
\pm 0,0675\end{array}$ & $\begin{array}{c}0,8422 \\
\pm 0,0257\end{array}$ & - & & & \\
\hline $\mathrm{h}_{4}$ & $\begin{array}{c}0,4228 \\
\pm 0,0847\end{array}$ & $\begin{array}{c}0,7173 \\
\pm 0,0421\end{array}$ & $\begin{array}{c}0,9114 \\
\pm 0,0110\end{array}$ & - & & \\
\hline $\mathrm{h}_{5}$ & $\begin{array}{c}0,2876 \\
\pm 0,0983\end{array}$ & $\begin{array}{c}0,5884 \\
\pm 0,0577\end{array}$ & $\begin{array}{c}0,8263 \\
\pm 0,0249\end{array}$ & $\begin{array}{c}0,9329 \\
\pm 0,0103\end{array}$ & - & \\
\hline $\mathrm{h}_{6}$ & $\begin{array}{c}0,1811 \\
\pm 0,1040\end{array}$ & $\begin{array}{c}0,4882 \\
\pm 0,0652\end{array}$ & $\begin{array}{c}0,7424 \\
\pm 0,0369\end{array}$ & $\begin{array}{c}0,8669 \\
\pm 0,0218\end{array}$ & $\begin{array}{c}0,9495 \\
\pm 0,0085\end{array}$ & \\
\hline
\end{tabular}

ee: Error Estándar

La $r_{\text {fenotípica }}$ también sufre una disminución monótona de 0,88 a 0,18 desde $h_{1}{ }^{*} h_{2}$ a $h_{1}{ }^{*} h_{6}$. Esta situación junto con la expresada en los dos cuadros anteriores confirma una tendencia hacia una correlación edad-edad muy baja entre plantas y árboles adultos.

\section{-Nothofagus obliqua}

En el Cuadro $\mathrm{N}^{\circ} 11$ se muestra las heredabilidades en sentido restringido o individual para cada una de las seis mediciones de altura de las progenies de $N$. obliqua.

\section{Cuadro $\mathrm{N}^{\circ} 11$}

$H^{2}$ PARA CADA UNA DE LAS SEIS MEDICIONES DE ALTURA DE LAS PROGENIES DE N. obliqua, EN CONDICIONES DE INVERNADERO

\begin{tabular}{|c|c|}
\hline Rasgos & Heredabilidad Individual \pm ee \\
\hline$h^{2}{ }_{1}$ & $0,4053 \pm 0,0599$ \\
\hline$h^{2} h 2$ & $0,7032 \pm 0,0806$ \\
\hline$h^{2}{ }_{h 3}$ & $0,6567 \pm 0,0802$ \\
\hline$h^{2}{ }^{2}$ & $0,5489 \pm 0,0774$ \\
\hline$h_{h 5}^{2}$ & $0,4291 \pm 0,0697$ \\
\hline$h_{h 6}^{2}$ & $0,4982 \pm 0,0749$ \\
\hline
\end{tabular}

ee: Error Estándar 
La $\mathrm{h}^{2}$ individual para las mediciones secuenciales de altura de las plántulas de $N$. obliqua muestran un importante control genético con errores estándares pequeños, con valores que fluctúan de 0,40 a 0,49 para $h_{h 1}^{2}$ y h$^{2}{ }_{h 6}$ respectivamente, encontrándose valores tan alto como de 0,70 para $h^{2}$ h2. Los altos valores observados obedecen, entre otras causas, a la escasa variación ambiental existente dentro del invernadero, de modo que gran parte de las diferencias observadas obedecen a variación de carácter genético. En el Cuadro $N^{\circ} 12$ se muestra la correlación genética entre las seis mediciones de altura de la progenie de $N$. obliqua.

\section{Cuadro $\mathrm{N}^{\circ} 12$}

CORRELACIONES GENÉTICAS ENTRE MEDICIONES DE ALTURA DE LAS PROGENIES DE $N$. obliqua EN CONDICIONES DE INVERNADERO

\begin{tabular}{|c|c|c|c|c|c|c|}
\hline $\begin{array}{c}\mathbf{r}_{\text {genética }} \\
(x, y)\end{array}$ & $h_{1} \pm e e$ & $h_{2} \pm$ ee & $h_{3} \pm$ ee & $h_{4} \pm$ ee & $h_{5} \pm e e$ & $h_{6} \pm e e$ \\
\hline $\mathrm{h}_{1}$ & - & & & & & \\
\hline $\mathrm{h}_{2}$ & $\begin{array}{c}0,9331 \\
\pm 0,0247\end{array}$ & - & & & & \\
\hline $\mathrm{h}_{3}$ & $\begin{array}{c}0,8527 \\
\pm 0,0366 \\
\end{array}$ & $\begin{array}{c}0,9450 \\
\pm 0,0119 \\
\end{array}$ & - & & & \\
\hline $\mathrm{h}_{4}$ & $\begin{array}{c}0,7012 \\
\pm 0,0553 \\
\end{array}$ & $\begin{array}{c}0,7929 \\
\pm 0,0339 \\
\end{array}$ & $\begin{array}{c}0,8950 \\
\pm 0,0185 \\
\end{array}$ & - & & \\
\hline$h_{5}$ & $\begin{array}{c}0,5962 \\
\pm 0,0682\end{array}$ & $\begin{array}{c}0,6692 \\
\pm 0,0505\end{array}$ & $\begin{array}{r}0,7645 \\
\pm 0,0382\end{array}$ & $\begin{array}{c}0,9361 \\
\pm 0,0134\end{array}$ & - & \\
\hline $\mathrm{h}_{6}$ & $\begin{array}{c}0,5192 \\
\pm 0,0747\end{array}$ & $\begin{array}{c}0,5701 \\
\pm 0,0605\end{array}$ & $\begin{array}{c}0,6668 \\
\pm 0,0497\end{array}$ & $\begin{array}{c}0,8460 \\
\pm 0,0268\end{array}$ & $\begin{array}{c}0,9334 \\
\pm 0,0133\end{array}$ & - \\
\hline
\end{tabular}

ee: Error Estándar

La $r_{\text {genética }}$ entre las mediciones desciende desde $0,93\left(h_{1}{ }^{*} h_{2}\right)$ a $0,51\left(h_{1}{ }^{*} h_{6}\right)$, desde la primera medición a la sexta medición. La correlación edad-edad es ligeramente mejor que para $N$. alpina, aunque también experimenta una disminución en la medida que se comparan mediciones más distantes.

\section{Cuadro $\mathrm{N}^{\circ} 13$}

\section{CORRELACIONES FENOTÍPICA ENTRE LAS MEDICIONES DE ALTURA DE LAS PROGENIES DE $N$. obliqua EN CONDICIONES DE INVERNADERO}

\begin{tabular}{|c|c|c|c|c|c|c|}
\hline $\begin{array}{c}\mathbf{r}_{\text {fenotípica }} \\
(\mathbf{x}, \mathbf{y})\end{array}$ & $\mathbf{h}_{\mathbf{1}} \pm \mathbf{e e}$ & $\mathbf{h}_{\mathbf{2}} \pm \mathbf{e e}$ & $\mathbf{h}_{\mathbf{3}} \pm \mathbf{e e}$ & $\mathbf{h}_{\mathbf{4}} \pm \mathbf{e e}$ & $\mathbf{h}_{\mathbf{5}} \pm \mathbf{e e}$ & $\mathbf{h}_{\mathbf{6}} \pm \mathbf{e e}$ \\
\hline $\mathrm{h}_{1}$ & - & & & & & \\
\hline $\mathrm{h}_{2}$ & $\begin{array}{c}0,6515 \\
\pm 0,0250\end{array}$ & - & & & & \\
\hline $\mathrm{h}_{3}$ & 0,5309 & 0,8617 & - & & & \\
& $\pm 0,0371$ & $\pm 0,0108$ & & & & \\
\hline $\mathrm{h}_{4}$ & 0,3924 & 0,7172 & 0,8566 & - & & \\
& $\pm 0,0519$ & $\pm 0,0266$ & $\pm 0,0146$ & & & \\
\hline \multirow{2}{*}{$h_{5}$} & 0,3044 & 0,6173 & 0,7556 & 0,9025 & & \\
\hline \multirow{2}{*}{$h_{6}$} & 0,0593 & $\pm 0,0345$ & $\pm 0,0233$ & $\pm 0,0103$ & & \\
\hline
\end{tabular}

ee: Error Estándar 
Considerando que las mediciones consideradas fueron efectuadas aproximadamente cada 15 días, también se espera una correlación muy baja entre árboles adultos y plantas. Finalmente, la $r_{\text {fenotípica }}$ es alta para $h_{1}{ }^{*} h_{2}$ de 0,65 y desciende a 0,16 para $h_{1}{ }^{*} h_{6}$. En el Cuadro $N^{\circ}$ 13 se muestra la correlación fenotípica entre las seis mediciones de altura de la progenie de $N$. obliqua.

\section{DISCUSIÓN Y CONCLUSIONES}

Los Nothofagus son arboles polinizados por el viento, es decir polinización anemófila. El control genético del tamaño de la semilla es potencialmente determinado por tres componentes genéticos; la planta polinizadora, la planta que porta la semilla y la constitución genética del embrión (Silvertown y Lovett-Doust, 1993).

La heredabilidad clonal para el largo y peso de la semillaes alta para ambas especies de Nothofagus, no obstante los valores son superiores para $N$. obliqua $(0,5785 \pm 0,0197)$, incluso su error estándar es menor. Estos valores son superiores a los obtenidos por Chaisurisri et al 1992 en Picea sitchensis, que alcanza una heredabilidad moderada en sentido amplio para el peso de la semilla (0,36). Sorensen y Campbell (1993), sugieren que las semillas más pesadas de Pseudotsuga menziessi var. Menziesii (Mirb.) Franco producen plantas más altas a los dos años que semillas más ligeras.

Donoso (1979a; 1979b) estableció la existencia de una gradiente clinal del tamaño de la semilla, entre otros. La comprobación de un nivel de fijación genética importante para ambos rasgos estudiados aporta antecedentes para entender la dinámica de la estructura genética de esta especie y abre algunas puertas sobre la utilización de la variación genética.

La evaluación temprana de las características de crecimiento de las plantas bajo condiciones controladas permite obtener valores más depurados, en el sentido que han estado por menos tiempo influenciadas por efectos ambientales acumulativos, de esta forma la variación expresada corresponde en mayor medida a efectos genéticos.

Lo anterior se confirma también en los altos niveles de heredabilidad estimados para características de capacidad germinativa y crecimiento inicial, tanto en $N$. obliqua como en $N$. alpina. No obstante, es destacable la correlación negativa entre la energía germinativa y, los parámetros capacidad y valor de germinación. El análisis de germinación fue llevado en condiciones de laboratorio, donde la variabilidad ambiental durante el ensayo de germinación es muy escasa, y los estudios de crecimiento se han evaluado en condiciones controladas de invernadero, donde dichas condiciones han sido iguales para todas las procedencias ensayadas. Este alto control de las condiciones ambientales determina que las estimaciones de parámetros genéticos sean muy precisas. En un programa de mejora genética de mediano y largo plazo es muy importante hacer estos análisis ya que sus resultados son muy orientadores respecto del potencial genético de los arboles, las familias y procedencias en el futuro, en especial hoy en día, donde es necesario hacer un seguimiento de la presión de selección que está ejerciendo el cambio climático.

También se observa heredabilidades altas para características como el numero de semillas por kilo, por su parte el peso de las semillas individuales exhibe heredabilidades entre arboles un poco menores, como consecuencia de su mayor dependencia de efectos ambientales externos, en este sentido cabe mencionar que tanto la semilla de $N$. obliqua como de $N$. alpina presenta perforaciones provocadas por el ataque del microlepidóptero Perzelia spp. Este insecto en su etapa larval perfora los frutos y devora parcial o totalmente el embrión de las semillas. La 
incidencia del daño es distinta en $N$. obliqua que en $N$. alpina, en la primera especie el porcentaje de perforaciones disminuye de norte a sur, mientras que en $N$. alpina el fenómeno se acentúa con la latitud.

Las variables de crecimiento inicial en altura tienen una alta correlación entre sí, tanto genética como fenotípica, aún así la tendencia es que los valores de correlación disminuyan en función del tiempo transcurrido entre cada par de mediciones. La tasa de disminución de la correlación sugiere una baja correlación entre plantas y arboles adultos, a menos que en un momento determinado las correlaciones edad - edad se estabilicen.

El importante control genético encontrado en los rasgos estudiados indica claramente una fijación alelica de dichas características, lo que unido a la variación de dichos rasgos en función de gradientes ambientales, permite orientar el ordenamiento y la estrategia de mejora genética para estas especies de Nothofagus. En definitiva orienta a cómo utilizar la variación genética para un uso sostenible.

\section{REFERENCIAS}

Burdon, R.; Bannister, M. y Low, C., 1992. Genetic Survey of Pinus radiata. 4: Variance Structure and Heritabilities In Juvenile Clones. New Zealand Journal of Forestry Science 22 (2/3): 187-210.

Chaisurisri, K; Edwards, G. y El-Kassaby, Y., 1992. Genetic Control of Seed Size and Germination in Sitka Spruce. Silvae Genetica. 41, 6:348-355.

Donoso, C., 1979a. Genecological Differentiation in Nothofagus obliqua (Mirb.) Oerst. in Chile. Forest Ecology and Management 2:53-66.

Donoso, C., 1979b. Variación y Tipos de Diferenciación en Poblaciones de Roble (Nothofagus obliqua (Mirb.) Oerst). Bosque 3(1)1-14.

Donoso, C., 1993. Bosques Templados de Chile y Argentina, Variación y Estructura Dinámica. Editorial Universitaria. Chile. 483 P.

Donoso, C., 1996. Ecology of Nothofagus Forests in Central Chile. En: The Ecology and Biogeography of Nothofagus Forest. Edited by Thomas T. Veblen, Robert S. Hill, and Jennifer Read. Yale University Press, New Haven and London, Pp. 271-292

El-Kassaby, Y; Edwards, G. y Taylor, D., 1992. Genetic Control of Germination Parameters in Douglas-Fir and its Importance for Domestication. Silvae Genetica 41, 1:48-54.

Fuenzalida, H., 1965. Clima, En: Geografía Económica de Chile. Texto Refundido. Corporación de Fomento de la Producción (CORFO). Santiago. Pp: 99-152

Gilmour, A; Cullis, B; Welham, S. y Thompson, R., 2000. ASREML Reference Manual. Version Beta. Nsw Agriculture. $227 \mathrm{P}$.

Hartmann, H. y Kester, D., 1975. Plant Propagation: Principles and Practices. Prentice Hall. 880 P.

Ipinza, R; Garcia, X.; Apiolaza, L.; Molina, M.; Chung, P. y Parra, P., 1994. Variación Juvenil de un Ensayo de Procedencias y Familias De Eucalyptus globulus subsp. globulus Labill., en la Séptima Región, Chile. Ecología No. 8, Pp: 259-270.

ISTA, 1993. Reglas Internacionales para el Ensayo de Semilla. 3 Ed. Editorial Rabasa SA. México, 24(3):127 P.

Medina, A., 2000. Determinación del Grado de Control Genético en Parámetros de Calidad y Forma de la Semilla, Parámetros Germinativos y Variables de Crecimiento Inicial para Procedencias de Nothofagus alpina (Poepp. et Endl) Oerst. Tesis De Grado. Facultad de Ciencias Forestales. Universidad Austral de Chile. 
Ordóñez, A., 1986. Germinación de las Tres Especies de Nothofagus Siempreverdes (Coigües) y Variabilidad en la Germinación de Procedencias de Coigüe Común ( $N$. dombeyi). Facultad de Ciencias Forestales. Universidad Austral de Chile.

Rodriguez, J. y Medina, A., 2000. Viverizacion de Procedencias de N. alpina y N. obliqua en Contenedores. En: Domesticación y Mejora Genética de Raulí y Roble (Ed. Ipinza, R; Gutierrez, B. y Emhart, V.). 307-347.

Silverston, J. y Lovett-Doust, J., 1993. Introduction to Plant Population Biology. Blackwell Scientific Publications, Oxford, Uk. 244 P.

Sorensen, F. y Campbell, R., 1993. Seed Weight - Seedling Size Correlation in Coastal Douglas-Fir: Genetic and Environmental Components. Can. J. For. Res. 23:275-285.

Vergara, R.; Ipinza, R.; Donoso, C. y Grosse, H., 1998. Definición de Zonas de Procedencias de Roble y Rauli. Estado de Avance. En: Primer Congreso Latinoamericano IUFRO, 'El Manejo Sustentable de los Recursos Forestales: Desafíos del Siglo XXI". Valdivia, Noviembre de 1998. 